\title{
LAS PTERIDOFITAS EN LA HISTORIA DE LAS PLANTAS DE LA NUEVA ESPAÑA DE FRANCISCO HERNANDEZ, PROTOMEDICO ESPAÑOL
}

\author{
Ramon Riba, Blanca Perez-Garcia \\ Universidad Autónoma Metropolitana-Iztapalapa \\ Depto. de Biología, C.B.S. \\ Apdo. Postal 55-535 \\ 09340 México, D.F. \\ $Y$ \\ Alma Orozco Segovia \\ Centro de Ecología, UNAM \\ Apdo. Postal 70-275 \\ 04510 México, D.F.
}

\begin{abstract}
RESUMEN
En la Historia de las Plantas de la Nueva España, Francisco Hemández (1517/1518-1584) incluye 3076 plantas, mencionadas con sus nombres indígenas además de describirlas brevemente $y$ de indicar sus usos en la medicina nativa y el lugar en que crecen; algunas de ellas las compara con otras plantas, americanas o europeas. Cincuenta y dos las señala como "helechos" de manera directa o indirecta pero lamentablemente, sólo ilustra cinco de ellas. Estudios previos sobre esta obra de Hernández hechos por F. Altamirano, M. Altamirano, M. A. Batalla et al., F. Miranda y J. Valdés, F. Miranda et al. y J. Valdés y H. Flores tuvieron como objeto la identificación botánica de las plantas de la obra, incluyendo las pteridofitas, pero por la vaguedad de las descripciones, tuvieron éxito parcial. En este trabajo y con base en los estudios anteriores, se presenta un nuevo intento de interpretación de las pteridofitas incluidas en la obra de Hemández. De las cincuenta y dos pteridofitas mencionadas antes, a nuestro juicio y por datos mencionados en las descripciones, cinco son angiospermas (dos ranunculáceas, una piperácea, una leguminosa o coriariácea y una de familia desconocida); doce más, aunque sí parecen ser pteridofitas, no es posible identificarlas con los datos aportados en las descripciones. Las treinta y cinco plantas restantes permiten su identificación con cierta seguridad, por lo menos a nivel genérico.
\end{abstract}

\section{ABSTRACT}

Francisco Hemández (1517/1518-1584), in his Historia de las Plantas de la Nueva España, included 3076 plants with native names, descriptions, medicinal uses and region where they grow. Fifty two of them have been identified as fems but unfortunately only five are illustrated. Previous studies of the books of Hemández were made by F. Altamirano, M. Altamirano, M. A. Batalla et al., F. Miranda and J. Valdés, F. Miranda et al., and J. Valdés and H. Flores, who attempted the botanical interpretation of the plants with partial success, because of the vagueness of the descriptions. Based on the former interpretations, a new evaluation of the pteridophytes included in Hemández is presented. We believe that five plants of the fifty two pteridophytes mentioned are angiosperms (2 Ranunculaceae, 1 Piperaceae, 1 Leguminosae or Coriariaceae and 1 of unknown family); the interpretation of twelve plants 
is not possible, although apparently they are pteridophytes. The remaining 35 plants can be interpreted as pteridophytes, at least at the genus level.

\section{INTRODUCCION}

Francisco Hernández, nombrado por Felipe II "Protomédico de las Indias, Islas y tierra firme del Mar Océano", viaja al nuevo continente en 1570 para conocer sus productos naturales. Entre 1571 y 1576 recorre distintas regiones de Nueva España, sobre todo por el Valle de México y el actual estado de Morelos, estudiando la flora y la fauna de la zona; por el sur hasta las costas de Oaxaca y Guerrero, a Veracruz por el este y recorre algunas localidades como Jiquilpan, Cuitzeo y Pátzcuaro entre otras, por el oeste. Lamentablemente, la totalidad de sus viajes no se conoce con certeza, al igual que una gran parte de su obra (para más datos acerca de la vida y la obra de F. Hernández, véase Somolinos D'Ardois, 1960). En estos viajes Hernández recogió una gran cantidad de información acerca de, entre otras cosas, la herbolaria nativa y, en los documentos que han sido hallados por los estudiosos, están contenidos valiosísimos datos acerca de las reales o supuestas propiedades medicinales de 3076 plantas utilizadas por los nativos.

$\mathrm{Ya}$ anteriormente otros investigadores han intentado la interpretación botánica del documento con relativo éxito (véase bibliografía al final del texto, especialmente Flores Olvera \& Valdés Gutiérrez, 1979). Para este trabajo se han revisado los datos referentes a cincuenta y dos plantas utilizadas en la herbolaria indígena y a las que Hernández explícitamente llama "helechos" o que suponemos que lo son, por la descripción que se ofrece o por los nombres comunes que se les daban a plantas similares en España. Su interpretación es difícil por varias razones. Hernández compara a algunos helechos de la Nueva España con los europeos y, aunque en algunos casos esto es acertado (p.e. en especies del género Adiantum), en otros no es así. Lo anterior sucede con los helechos a los que Hernández llama "lengua de ciervo" los que, por la descripción dada bajo este nombre en el Diccionario de la Lengua Española de la Real Academia (1941), son aspleniáceos de hoja simple (Phyllitis), pero Hernández amplía el término a los que creemos son otros géneros como Elaphoglossum, Microgramma o Pleopeltis. Además, las descripciones son poco precisas y de las representaciones gráficas solamente se han conservado cinco y, de ellas, una no muestra a un helecho, sino probablemente a una leguminosa o coriariácea.

\section{METODO}

1. Se revisaron las descripciones de las 3076 plantas comprendidas en la obra, seleccionando, para fines de este trabajo:

a) aquellas que, Hernández explícitamente llama "helechos".

b) aquellas que, por rasgos expresados en las descripciones, consideramos que pudieran ser pteridofitas (incluyendo helechos y plantas afines).

c) las que, por su nombre local (según Hernández), suponemos que puedan ser pteridofitas. 
2. Esta información se confrontó con las interpretaciones dadas por otros autores, cuando las hubiere.

3. Con lo anterior como punto de partida, se procedió a revisar ejemplares de herbario de la colección de pteridofitas de MEXU y de UAMIZ.

4. Con base en el grado de concordancia de las descripciones de Hernández con las características distintivas de las muestras de herbario seleccionadas y de su área de distribución, con la opinión de autores previos y la propia, se llegó a la decisión correspondiente.

Se tomó en cuenta que el nombre local en el lenguaje de la región es de importancia, ya que puede ser indicativo de las propiedades curativas de la planta, del hábitat o de su aspecto (hábito) pero, por desgracia, el nombre indígena en muchos casos ya se ha perdido en el transcurso del tiempo; además, tal nombre pocas veces se encuentra registrado en las etiquetas de los ejemplares de herbario, por lo que esta fuente no nos fue de mucha utilidad, salvo en contados casos.

\title{
RESULTADOS
}

La relación de las plantas incluidas en esta revisión se presenta a continuación de la siguiente manera:

a) Pteridofitas identificables por lo menos a género.

b) Plantas que, a juicio de los autores, no son pteridofitas.

c) Pteridofitas no identificables

En la referencia de cada planta se incluye:

a) Ubicación en la obra (p. ej. 3-1l = libro 3-capítulo 2).

b) Nombre en lenguaje nativo.

c) Nombre en español.

d) Opinión de otros autores (cuando existe).

e) Interpretación en el presente trabajo.

\section{PTERIDOFITAS IDENTIFICABLES A GENERO}

\author{
1-CXV \\ Del ACATZANAÍCXITL \\ $\circ$ pie de estornino
}

Otros autores: En Anónimo (PI. Mex., s. fecha) se interpreta como Adianthum (sic). Paso y Troncoso (1886) lo interpreta como Equisetum. Batalla et al. (1942-1943) lo interpretan como Pellaea ternifolia. 


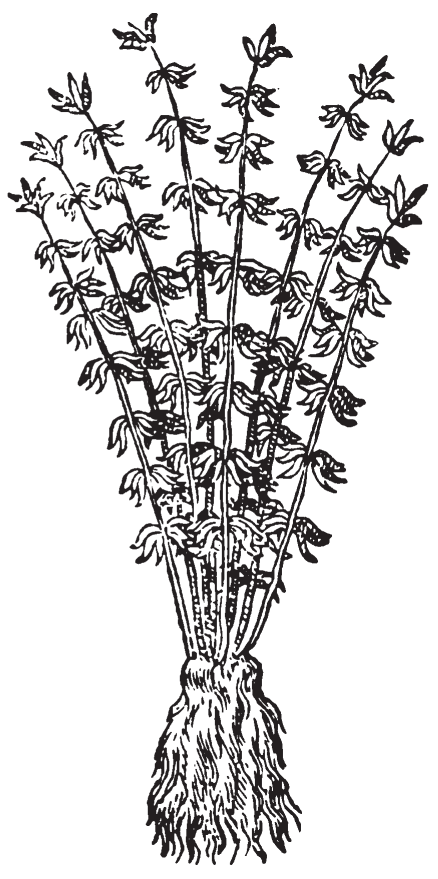

ACATZANAÍCXITL

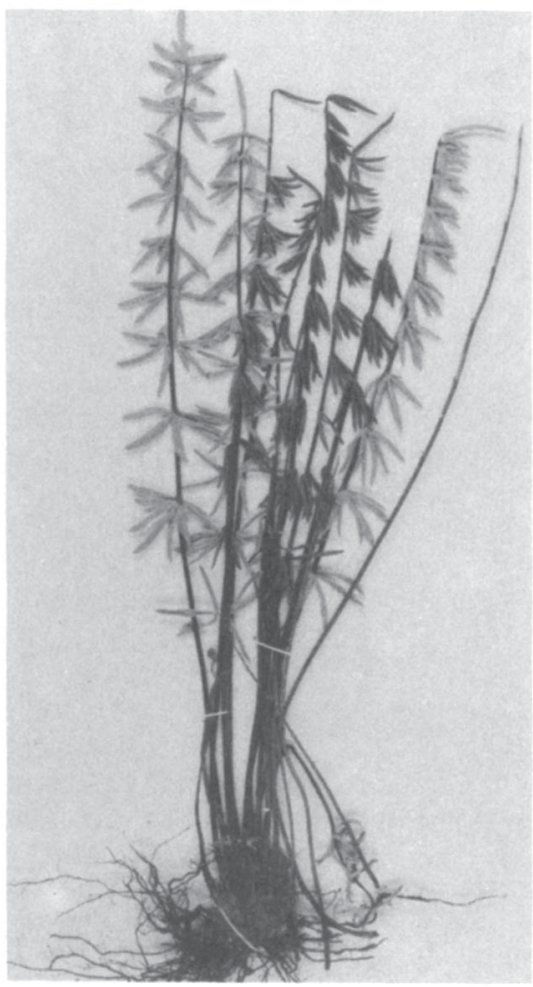

Fig. 1. Izq.: 1-CXV ACATZanaiCXITL o pie de estomino. Der.: Pellaea ternifolia (Cav.) Link.

Presente trabajo: Confirmamos la interpretación de Batalla et al. como Pellaea ternifolia (Cav.) Link. La ilustración de Hernández muestra con claridad la razón del epiteto especifico que se refiere a las pinnas divididas en tres segmentos, lo que permite la interpretación correcta de esta especie. Nótese la similitud de la ilustración de Hernández con la fotografia del ejemplar de herbario. (Fig. 1).

\section{$2-11$}

Del TLALAHOÉHOETL

$O$ abeto infimo

Otros autores: Batalla et al. (1942-1943), indican que podria ser Selaginella rupestris.

Presente trabajo: Es una microfilofita del grupo de Selaginella rupestris (L.) Spring, pero la especie es difícil de precisar, pues las diferencias entre ellas se dan básicamente por rasgos que se pueden apreciar solamente con una lupa o, en ciertos casos, se requiere de un microscopio estereoscópico. 
Riba et al.: Las Pteridofitas en la Historia de las Plantas de la Nueva España

3-I

Del ATLIPOZONZAPITZÁHOAC

o espuma de agua tenuifolia

Otros autores: En Anónimo (PI. Mex., s. fecha) se interpreta como Filix. Batalla et al. (1942-1943) señalan que Filix es sinónimo de Cystopteris.

Presente trabajo: Hernández incluye a esta planta entre las llamadas "capilares" por los europeos, “...como el culantrillo de pozo, la lengua de ciervo, el helecho (?), el polipodio y otras semejantes". Es muy probable que se trate de Athyrium filix-femina (L.) Roth, por los rasgos morfológicos y por el hábitat, aunque desconcierta que se diga que "nace .......abrazado a los troncos o a las peñas.....", ya que esta especie es terrestre y crece en suelo muy somero.

\section{3-IV \\ Del COATLICÉOAL purgante \\ o sombra de culebra}

Otros autores: En Anónimo (PI. Mex., s. fecha), Anónimo (PI. Med., s. fecha) y Batalla et al. (1942-1943) indican que podría ser Adiantum. Los últimos autores lo interpretan como A. capillus-veneris L.

Presente trabajo: Confirmamos la interpretación de los autores de que se trata de Adiantum sp., por hojas pequeñas con una pequeña hendedura, como carcomidas en su parte superior, ("culantrillo de pozo"). Es aventurado afirmar que es $A$. capillus-veneris $L$., pues en México crecen especies de Adiantum similares a dicha especie como $A$. tenerum, $A$. andicola y $A$. princeps y que difieren entre sí por rasgos no expresados en la descripción.

\section{3-VI \\ Del CAPOLXÍHUITL}

Otros autores: En Anónimo (PI. Med., s. fecha) y Batalla et al. (1942-1943) no ofrecen ningún comentario; M. Altamirano (Anónimo, PI. Mex., s. fecha) y Miranda y Valdés (1964) afirman que es un Polypodium.

Presente trabajo: Es probable que se trate de Phanerophlebia sp., "por las hojas oblongas y aserradas, dispuestas en hilera a uno y otro lado, y parecidas por consiguiente a las del capolin o cerezo de Indias".

\section{3-VII \\ Del OCOPÉTLATL de QUAUHTLA}

Otros autores: No ofrecen ningún comentario.

Presente trabajo: Es probable que se trate de Blechnum occidentale L. o $B$. glandulosum Link, por las características señaladas en la descripción y de manera importante por ser "...un lonquite áspero menor" según Hernández (véase capítulo de Discusión). 


\author{
$3-1 X$ \\ DeI HUAQUILIZPATLI \\ o medicina de los extenuados
}

Otros autores: Batalla et al. (1942-1943) indican que podría ser Aneimia (sic).

Presente trabajo: Confirmamos la interpretación de Batalla et al. como Anemia sp., aunque Hernández habla "de flores y fruto verde y en espiga"; suponemos que se refiere a la parte fértil de la hoja dimorfa de alguna especie de Anemia, la que podría confundirse con la inflorescencia de algunas angiospermas herbáceas; por el comentario de que "...Parece pertenecer a las especies de lengua de ciervo,...", se podría aventurar que es A. phyllitidis (L.) Sw., la que tiene las pinnas enteras y lanceoladas.

\title{
3-X \\ Del IPEPÉCHTETL \\ o base de piedra
}

Otros autores: No ofrecen ninguna interpretación.

Presente trabajo: Puede tratarse de Asplenium sp., por sus hojas (pinnas) de forma romboidal y aserradas.

\section{$3-X \mid$}

Del INETENPOPOAYA

o emuntorio de la boca

Otros autores: No ofrecen ningún comentario.

Presente trabajo: Puede tratarse de Diplazium ternatum Liebm., por las caracteristicas de "...pedúnculos o ramas largas y delgadas con tres hojas de lengua de ciervo, aserradas y oblongas" y por la localidad ("Xicotépec") que, según el mapa de los viajes, corresponde al actual Xicotepec de Juárez, Pue., en donde crece esta especie, además de existir en otros lugares. (Fig. 2).

\section{3-XII \\ Del ITZTICPATLI \\ o medicina fría}

Otros autores: Batalla et al. (1942-1943) indican que por las características señaladas por Hernández puede suponerse que es un Asplenium.

Presente trabajo: Apoyamos esta interpretación como Asplenium sp. 


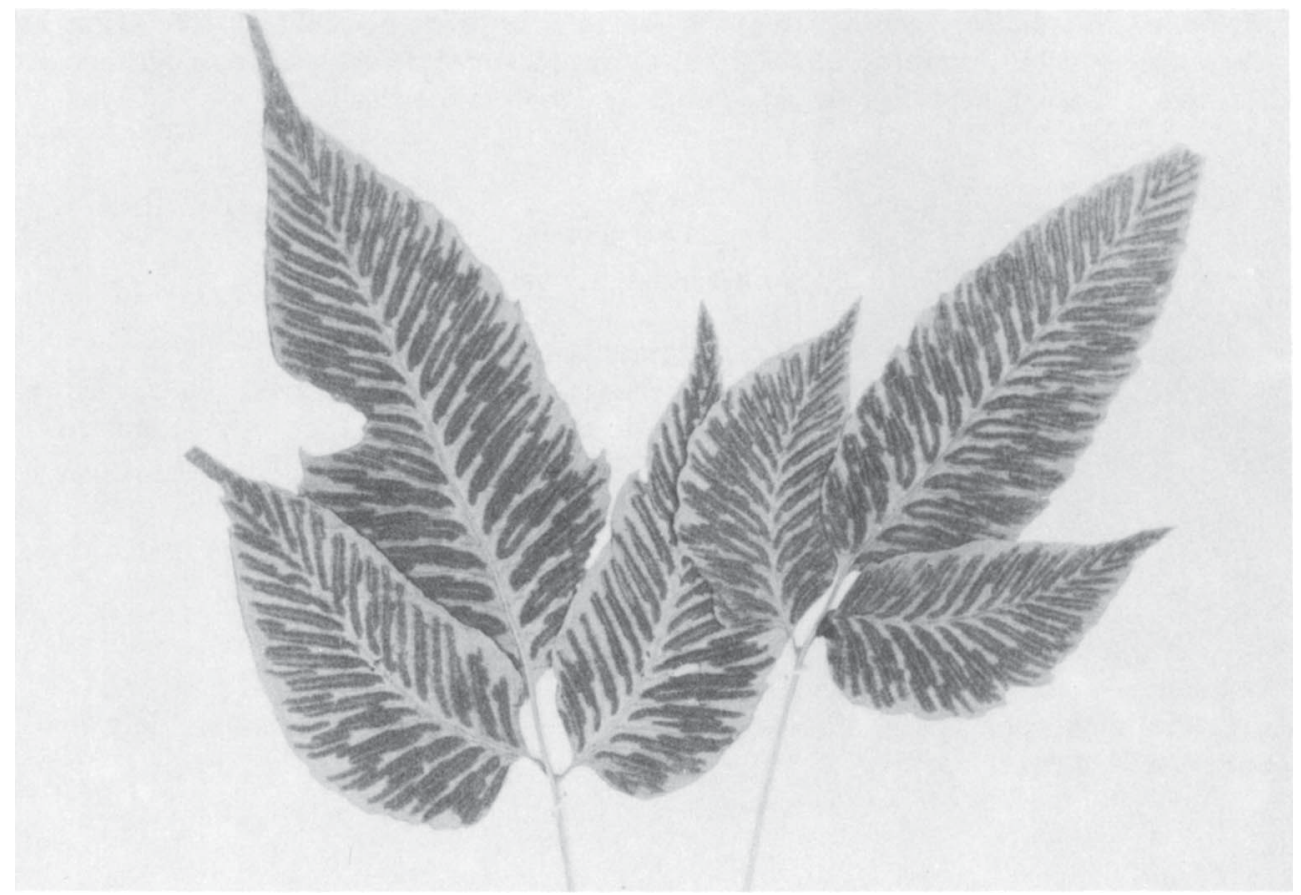

Fig. 2. Diplazium ternatum Liebm.

\author{
3-XIII \\ Del IMÁCPAL \\ o palma
}

Otros autores: Batalla et al. (1942-1943) informan que es un Adiantum basados en "raíces fibrosas y hojas parecidas a las del culantrillo".

Presente trabajo: Confirmamos la interpretación de Batalla et al., que se trata de Adiantum sp., aunque los datos contenidos en la descripción son escasos, además de que el nombre de "palma" se antoja raro para plantas del género Adiantum.

\title{
3-XIV \\ DeI ICXITECUAN \\ o pata de fiera
}

Otros autores: Batalla et al. (1942-1943), afirman que podría ser Phyllitis nigripes.

Presente trabajo: La descripción corresponde a Schaffneria nigripes Fée (sin.: Phyllitis nigripes (Fée) O. Ktze.), confirmando lo dicho por Batalla y colaboradores. Las características mencionadas en la descripción, "...hojas... de color verde oscuro, de mediano 
tamaño, casi redondas y con menudos dibujos de color pardo por debajo", esto último en referencia evidente a los soros dorsales y alargados, además de ubicarla entre las "lenguas de ciervo" (véase Discusión más adelante), confirman la identificación.

\author{
$3-X V$ \\ Del IZHOAYO \\ o medicina hojosa
}

Otros autores: No ofrecen ningún comentario.

Presente trabajo: Por las características dadas por Hernández es Asplenium serratum L., (“...muy semejante a la lengua de ciervo en toda su forma y naturaleza”).

\author{
3-XVI \\ Del YOHUALANPATLI \\ o medicina nocturna
}

Otros autores: No dan ningún comentario.

Presente trabajo: Por la similitud que hace Hernández con la "lengua de ciervo", puede ser Asplenium sp.

\author{
3-XVII \\ Del TECAPATLI \\ - medicina que nace junto a las corrientes de agua
}

Otros autores: No ofrecen ningún comentario.

Presente trabajo: Por las características señaladas por Hernández (...cierta especie de "lengua de ciervo"), hoja con puntos leonados por debajo y con tres puntas y dos senos es probable que sea Hemionitis sp.

\title{
3-XIX \\ Del OCOPÉTLATL HOAXTEPECENSE
}

Otros autores: Batalla et al. (1942-1943) afirman que es Adiantum sp.

Presente trabajo: Consideramos que es Adiantum concinnum Willd., por "ramitas dispuestas en hilera a uno y otro lado y llenas de hojas de culantrillo".

\section{3-XXIII}

Del OCOPETLATLPATLÁHUAC

o latifolio

Otros autores: Batalla et al. (1942-1943) comentan que Polypodium crassifolium y Campyloneurum phyllitidis tienen hojas semejantes a las descritas por Hernández. 
Riba et al.: Las Pteridofitas en la Historia de las Plantas de la Nueva España

Presente trabajo: Puede ser Niphidium crassifolium (L.) Lellinger o Campyloneurum phyllitidis (L.) Presl.

\author{
3-XXIV \\ Del OCOPETLATLQUALONI \\ o comestible
}

Otros autores: No ofrecen ningún comentario.

Presente trabajo: Es muy probable que se trate de Marattia weinmanniifolia Liebm. - M. laxa Kunze, por el color y consistencia de la base de las hojas que es comestible, el tipo de hojas y la distribución geográfica. En Oaxaca se conoce como "maíz del monte" (Martínez, 1979). La referencia que hace Hernández a "...hojas gruesas, cortas, blancas con rojo y parecidas hasta cierto punto a las del maguey...", nos hace pensar que se refiere a la gruesa base biauriculada de las hojas de Marattia spp., blancas en su interior y de color rojo oscuro en la superficie, las que tostadas y molidas son comidas como pinole en algunas partes del estado de Oaxaca; los "tallos de seis palmos de largo" pueden ser los largos pecíolos de las hojas 3-pinnatífidas, de aquí la referencia a que tienen "...hojas pequeñas (segmentos últimos) parecidas a las de leguminosas y dispuestas como las del helecho". (Fig. 3).

\title{
3-XXV \\ Del segundo OCOPETLATLPATLÁHUAC
}

Otros autores: No ofrecen ningún comentario.

Presente trabajo: Es probable que se trate de Asplenium spp. o de Diplazium spp. ("...es otra lengua de ciervo").

\section{3-XXVI \\ DeI POYOMATLI}

Otros autores: No hacen comentarios.

Presente trabajo: Corresponde a Nephrolepis occidentalis Kze., por la característica de raices fibrosas y tuberosas y porque las hojas jóvenes a menudo son rojizas.

\section{3-XXVIII \\ Del QUAUHACXÓYATL \\ o acxóyatl silvestre}

Otros autores: No ofrecen ningún comentario.

Presente trabajo: Es probable que sea una especie de Blechnum de hojas dimorfas, de pinnas fértiles angostas ("...como las que suelen tener los pinos"), sin embargo, las especies mexicanas de Blechnum no tienen puntos blancos en las hojas, como lo menciona 


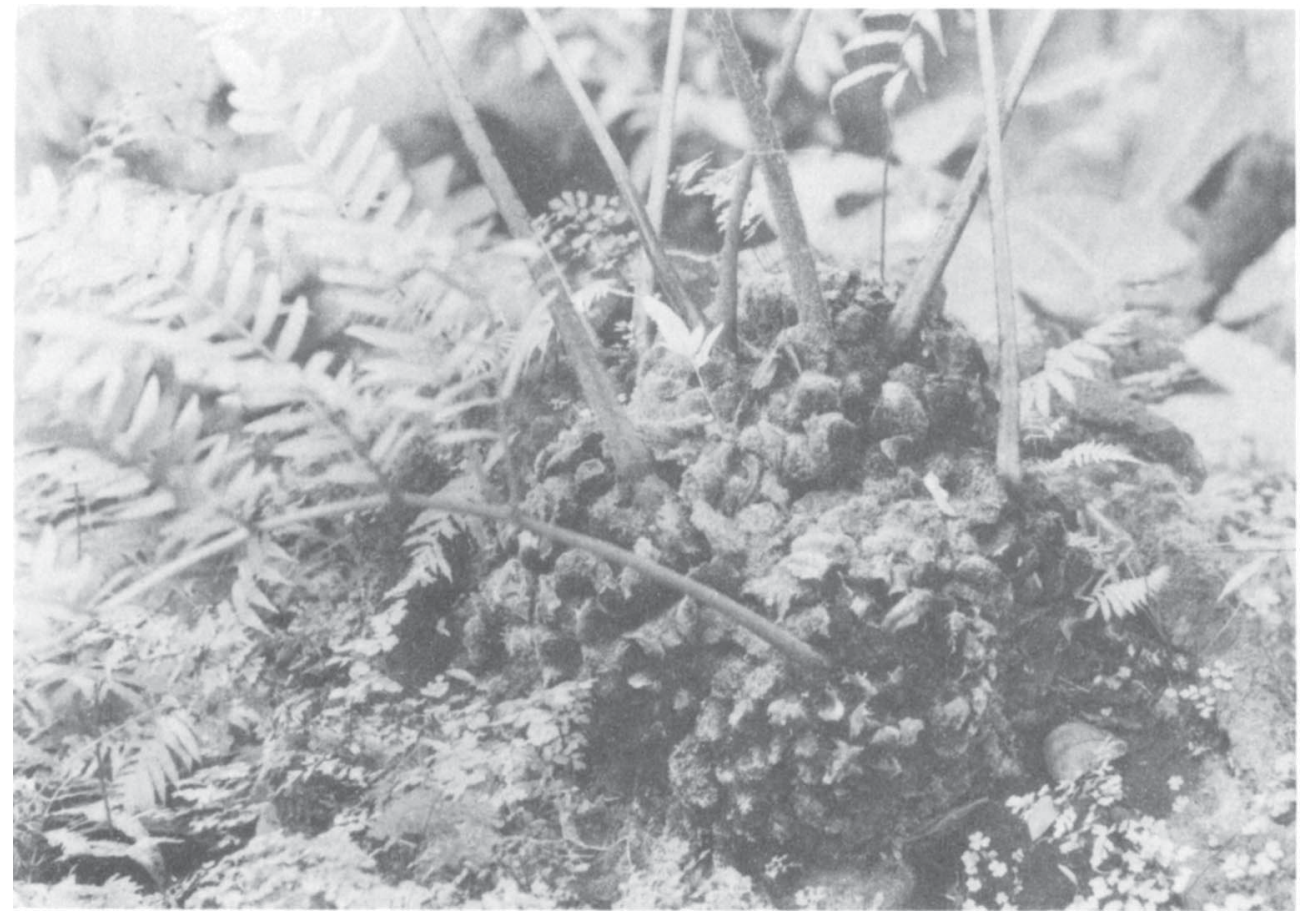

Fig. 3. Rizoma epigeo de Marattia laxa Kunze, mostrando las bases persistentes y auriculadas de las hojas. Fotografía de un ejemplar vivo en el Invernadero Faustino Miranda, Jardín Botánico de la UNAM.

Hernández, aunque los extremos de las venas son aparentes en algunas especies. El "color argénteo" del rizoma puede ser debido a la presencia de líquenes laminares.

\section{3-XXIX \\ DeI TLALQUEQUÉTZAL atataccense}

Otros autores: Batalla et al. (1942-1943) comentan que se trata de Polypodium lanceolatum.

Presente trabajo: Puede ser Pleopeltis sp. o Microgramma sp., por la descripción en que se menciona que es "...una hierbecilla de raíz transversal (rizoma?) cabelluda y parecida a la del polipodio...", además de que "...parece pertenecer a las variedades de lengua de ciervo" lo que seguramente hace referencia a que tiene las hojas simples. 
Riba et al.: Las Pteridofitas en la Historia de las Plantas de la Nueva España

\author{
$3-X X X I$ \\ Del segundo TEQUEQUÉTZAL
}

Otros autores: Batalla et al. (1942-1943) afirman que podría ser Adiantum.

Presente trabajo: Confirmamos que puede ser Adiantum ("culantrillo de pozo"), posiblemente Adiantum concinnum Willd., cuyas hojas jóvenes son rojizas ("...una cara de las hojas verde y la otra bermellón...").

\title{
3-XXXII \\ Del tercer TEQUEQUÉTZAL
}

Otros autores: No ofrecen ningún comentario.

Presente trabajo: Aunque con cierta reserva, es probable que se trate de Asplenium praemorsum Sw., pues Hernández la refiere a las lenguas de ciervo y señala que tiene "...hojas divididas en tres partes que terminan en punta", lo que hace pensar en las pinnas divididas de la especie mencionada.

\section{3-XXXIII \\ DeI TLATLACATON \\ o mascarilla}

Otros autores: Batalla et al. (1942-1943) afirman que es Asplenium sp.

Presente trabajo: El nombre vernáculo "lonquite" que le da Hernández a esta planta, se aplica en España a Blechnum spicant (L.) Roth y podría corresponder a tal especie "... de donde nacen tallos rojizos..., brillantes...", pero por la alusión a “...hojillas con forma de ala de murciélago", nos inclinamos a interpretar a esta planta como una especie 1-pinnada del género Asplenium.

\author{
3-XXXIV \\ Del TECUANIIZTE \\ ○ uña de fiera
}

Otros autores: Batalla et al. (1942-1943) consideran que es Adiantum sp.

Presente trabajo: Aunque la descripción no es definitiva, podría ser Adiantum sp.

\author{
3-XXXV \\ Del TECUANIIMA \\ o mano de fiera
}

Otros autores: No hacen ningún comentario.

Presente trabajo: Puede ser Tectaria heracleifolia (Willd.) Underw., con reservas. ("...cada tallo con tres hojas, oblongas, aserradas y sinuosas"). 


\section{3-XXXVII \\ Del TECUANIINÁCAZ \\ $u$ oreja de fiera}

Otros autores: No ofrecen ningún comentario.

Presente trabajo: Es probable que corresponda a Hemionitis sp. ("...hoja... y dividida con tres porciones redondeadas").

\section{3-XXXVIII \\ Del IMACPALTEPECUANI \\ pahuatlanense}

Otros autores: No hacen ningún comentario.

Presente trabajo: Interpretación muy dudosa; puede ser Lygodium sp., Doryopteris $\mathrm{sp}$., Hemionitis sp., por las características de "hojas sinuosas parecidas a una mano de fiera o como de vid".

\section{3-XXXIX \\ Del TANCÁPAZ \\ o helecho}

Otros autores: En Anónimo (PI. Med., s. fecha) se afirma que es polipodio. Batalla et al. (1942-1943) afirman que es Notholaena candida. (Fig. 4).

Presente trabajo: Es probable que corresponda a Aleuritopteris sp. o Notholaena sp.

\section{3-XL \\ DeI ITLANEXILLO \\ o pierna de liebre}

Otros autores: Paso y Troncoso (1886) señala que Hernández lo refiere a Adiantum. Batalla et al. (1942-1943) opinan que puede ser Pellaea cordata (Cav.) J. Smith.

Presente trabajo: Pellaea ovata (Desv.) Weatherby o especies afines ("...hojas pequeñas en forma de corazón").

6-LVI

Del CIHUAPATLI

mactlactlánico

Otros autores: Miranda et al. (1946) indican que es posiblemente Polypodium sp.

Presente trabajo: Puede corresponder a Elaphoglossum sp., (“...hojas.... por encima de un verde oscuro, amarillentos por debajo y con puntos rojos"). Algunas especies de 


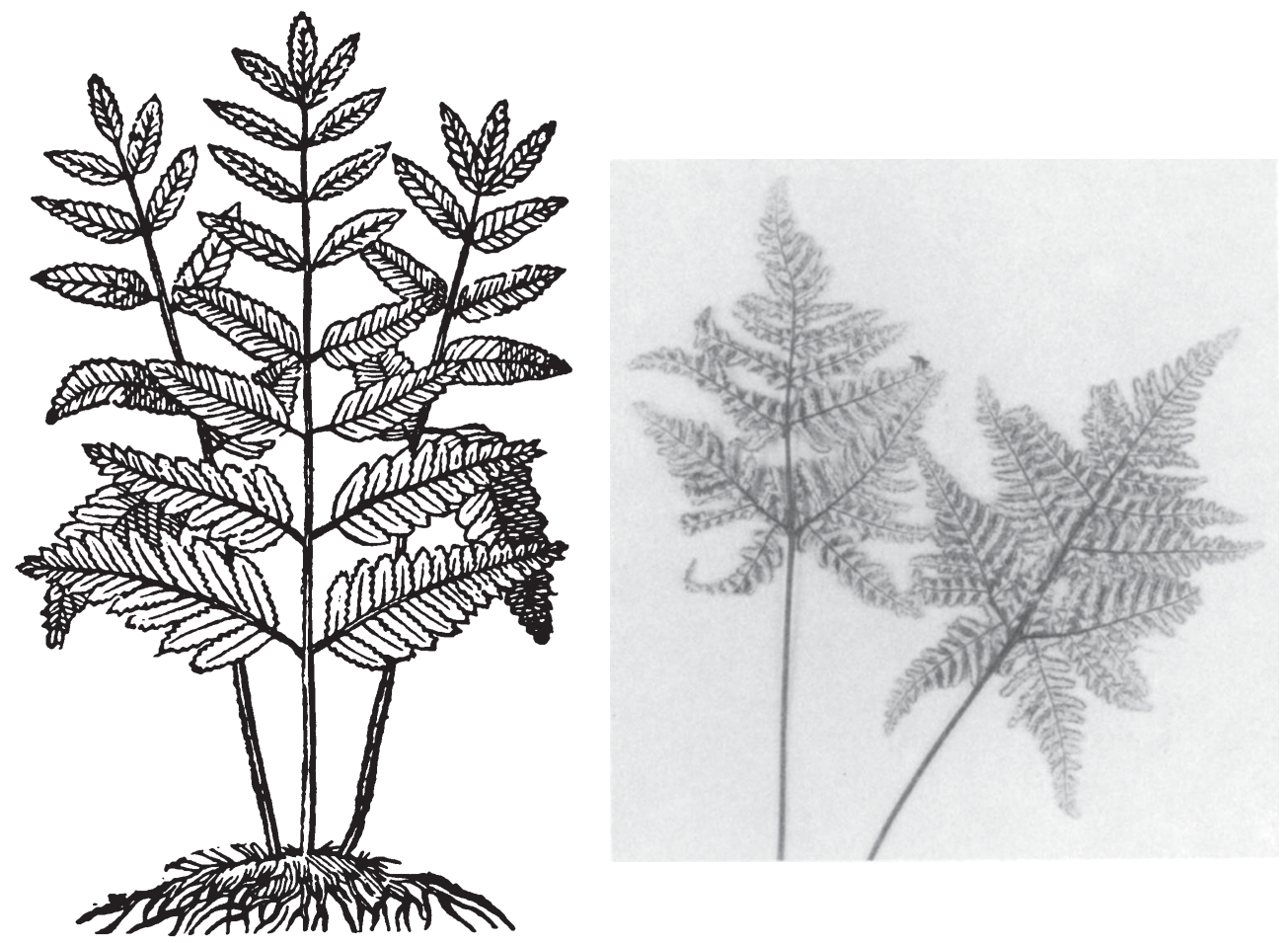

TANCÁPAZ

Fig. 4. Izq.: 3-XXXIX TANCÁPAZ o helecho. Der.: Cheilanthes hookeri (Küm.) Domin.

Elaphoglossum presentan el envés de la hoja fértil de color amarillo cuando las esporas no están maduras y los puntos rojos parecen corresponder a alguna secreción de la hoja en esta misma cara.

Aunque el nombre náhuatl "cihuapatli" (medicina de la mujer) le es dado a varias especies vegetales cuyas hojas en cocimiento son usadas para facilitar el parto, ninguna de ellas es una pteridofita y Hernández no menciona dicho uso de manera específica. 


\author{
6-CLIX \\ Del cuarto TEYYAUHTLI \\ que algunos llaman tzanaícxitl
}

Otros autores: Miranda et al. (1946) indican que el tricomanes de las farmacias es el helecho Asplenium trichomanes L. (Viejo Mundo).

Presente trabajo: Suponemos que es Asplenium sp., únicamente por la referencia que hace Hernández a que "...Parece ser de la misma forma y naturaleza del tricomanes...".

\title{
7-LXIX \\ Del QUAMIÁHOATL \\ o espiga de árbol
}

Otros autores: Miranda et al. (1946) afirman que puede ser Lycopodium dichotomum.

Presente trabajo: Muy similar a Lycopodium reflexum Lam., sobre todo por la raíz corta y ramificada (Fig. 5), los tallos dicotómicos erectos y las hojas delgadas, aunque el nombre "quamiáhoatl" o "espiga de árbol" hace pensar en una planta epífita por lo que podría corresponder a $L$. cuernavacense que crece en la región mencionada por Hernández; concordamos con Miranda en que no se le conocen aplicaciones. Aquí hay aparentemente una confusión, pues en una parte del texto de Hernández referente a esta planta, se dice que es semejante al "titímalo paralio" (Tithymalus paralius = Euphorbia paralias L., Euphorbiaceae) y los ejemplares de herbario de esta última son diferentes por completo de la planta descrita por Hernández.

\author{
21-XXXVII \\ Del CHARÁPETI \\ o planta escarlata apacincanense
}

Otros autores: En Anónimo (PI. Mex., s. fecha) se señala que es Rumex sanguineus y Beta vulgaris. Esta determinación es para otro "charápeti" (Libro IV, Cap. I). En PI. Med. (s. f.) se anota que es un helecho y también las especies citadas en PI. Mex., (s. f.).

Presente trabajo: Consideramos que las características pueden acercarnos a Pleopeltis sp. o a Elaphoglossum sp. (Fig. 6).

\section{PTERIDOFITAS NO IDENTIFICABLES}

\author{
1-XCIX \\ Del AHOYACPATLI \\ o medicina suave y olorosa
}

Otros autores: En Anónimo (PI. Mex., s. fecha), Anónimo (PI. Med., s. fecha) y Batalla et al. (1942-1943) es interpretado como Polypodium sp.

Presente trabajo: No es posible definir de qué planta se trata. 

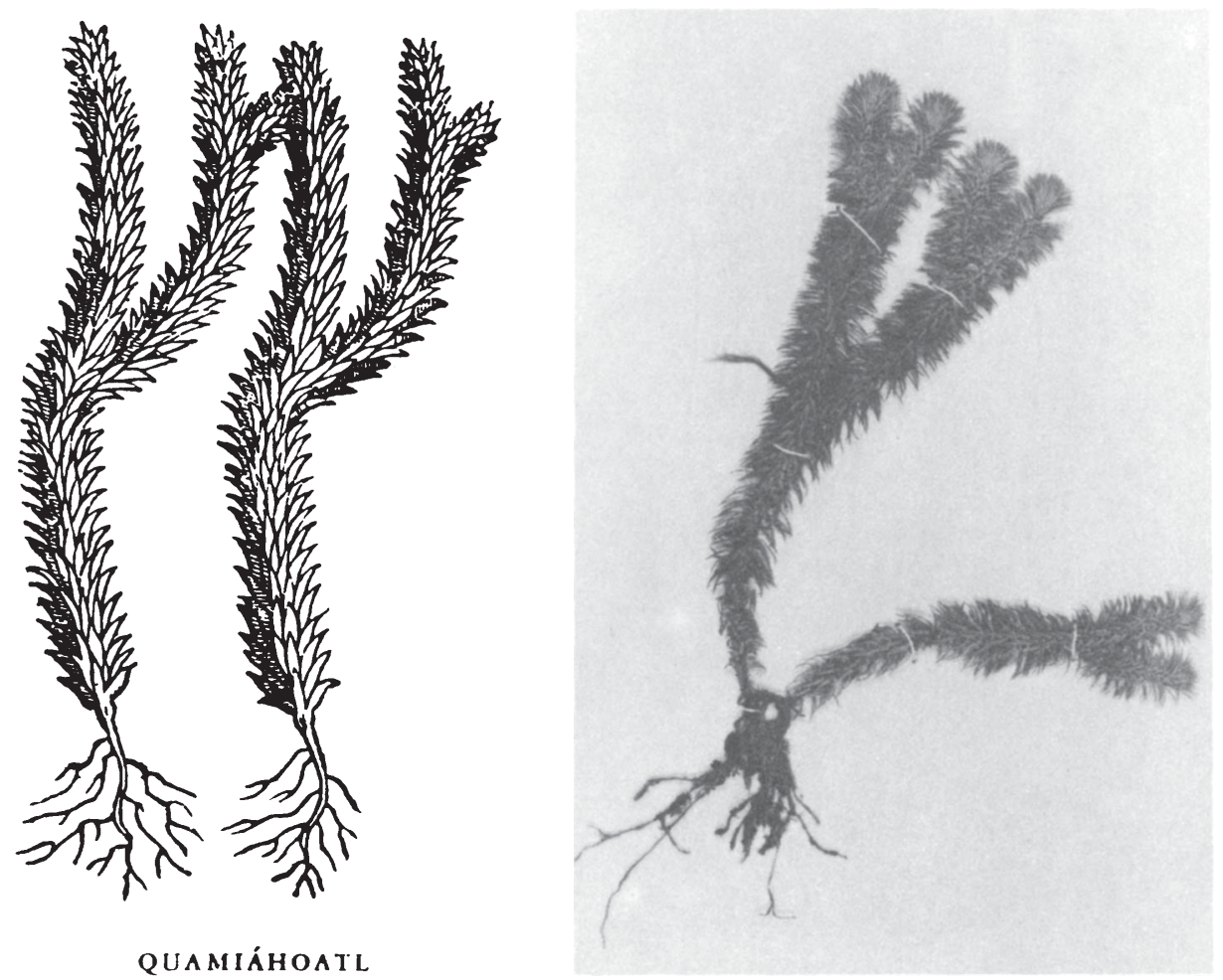

Fig. 5. Izq.: 7-LXIX QUAMIAHOATL o espiga de árbol. Der.: Lycopodium reflexum Lam.

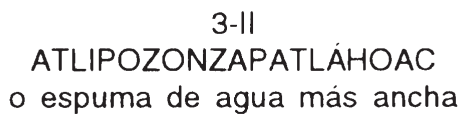

Otros autores: En Anónimo (PI. Mex., 1: 251 s. fecha), Anónimo (PI. Med., s. fecha) y Batalla et al. (1942-1943) se indica que es un Polypodium.

Presente trabajo: Los datos de la descripción son insuficientes. 

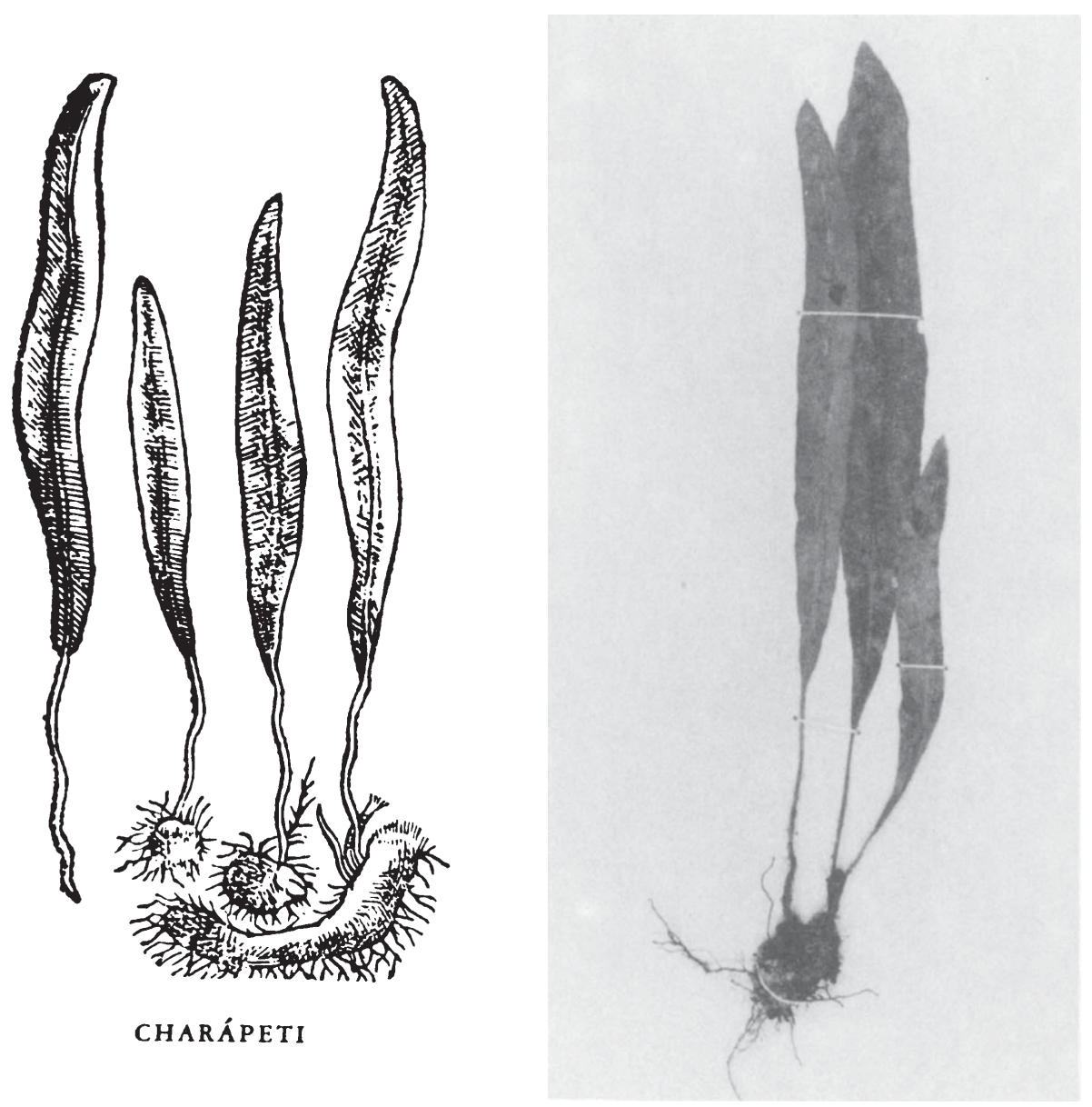

Fig. 6. Izq.: 21-XXXVII CHARÁPETI. Der.: Elaphoglossum sp.

\author{
3-III \\ AHOYACPATLI \\ o medicina suave
}

Otros autores: En Anónimo (PI. Mex., s. fecha), Anónimo (PI. Med., s. fecha) y Batalla et al. (1942-1943) indican que es un Polypodium. Paso y Troncoso (1886), no concreta la interpretación. 
Presente trabajo: Aunque es posible que el nombre "ocopetlatltepiton" con el que también era conocido, indique que se trate de un helecho, los datos son insuficientes para asegurar que es Polypodium sp.

\title{
3-V \\ COATLICÉOAL \\ pahuatlanense
}

Otros autores: No aportan información.

Presente trabajo: Parece tratarse de una planta parecida a un helecho ("...parecido al driópteris"), pero no es posible su interpretación.

\author{
3-VIII \\ ELMOYAHUIZPATLI \\ - medicina de la náusea
}

Otros autores: No aportan información.

Presente trabajo: Desconocido.

\section{$3-X^{*}$ \\ Del OCOPÉTLATL raro}

Otros autores: No aportan información.

Presente trabajo: * v. 3-XXII más adelante.

\author{
3-XXI* \\ Del segundo OCOPÉTLATL raro
}

Otros autores: No aportan información.

Presente trabajo: * v. 3-XXII más adelante.

\section{$3-X X 11^{*}$ \\ Del tercer OCOPÉTLATL raro}

Otros autores: No aportan información.

Presente trabajo: * Evidentemente, en estos tres casos se trata de helechos, pero los datos aportados por Hernández no permiten su interpretación. 


\title{
3-XXVII \\ Del QUIMICHPATLI \\ o medicina de ratón
}

Otros autores: No aportan información.

Presente trabajo: Es probable que se trate de una angiosperma.

\author{
$3-X X X$ \\ Del TEQUEQUÉTZAL \\ o cresta de piedra
}

Otros autores: No aportan información.

Presente trabajo: No es posible su interpretación.

\section{3-XXXVI \\ Del TECUANIIPÓPOL \\ o pelos de fiera}

Otros autores: No aportan información. interpretación.

Presente trabajo: Aunque es muy factible que sea un helecho, no es posible su

$$
16-L X X I 1
$$

Del QUAUHAMOXTLI

o musgo de los árboles

Otros autores: En Anónimo (PI. Mex., s. fecha), Anónimo (PI. Med., s. fecha) y Batalla et al. (1942-1943) es interpretado como Polypodium sp., calaguala o Tillandsia.

Presente trabajo: No es posible definir de qué planta se trata.

\section{ANGIOSPERMAS}

1-LVI

ATONAHUIZPATLI

o remedio de la fiebre

Otros autores: En Anónimo (PI. Mex., s. fecha) sugieren que es Ophyogloss (sic). Batalla et al. (1942-1943) no lo interpretan.

Presente trabajo: No es posible su interpretación, aunque por la descripción parece evidente que no es un helecho. 
Riba et al.: Las Pteridofitas en la Historia de las Plantas de la Nueva España

\author{
3-XVIII \\ OCOPÉTLATL \\ atataccense
}

Otros autores: No ofrecen ningún comentario.

Presente trabajo: Aunque Hernández dice que es "cierto género de helecho" y el nombre "ocopétlatl" se aplica en náhuatl a muchos de ellos, evidentemente hay una confusión, pues Hernández habla de "...flor y fruto dispuestos en espigas muy largas..." además de que la figura corresponde muy probablemente a una leguminosa herbácea o, en opinión de un revisor anónimo, podría ser Coriaria thymifolia.

\author{
$3-\mathrm{CXCl}$ \\ Segundo ATONAHUIZPATLI \\ de tepuzcululla
}

Otros autores: No ofrecen ningún comentario.

Presente trabajo: Por las características anotadas en la descripción, parece ser Peperomia sp.

\title{
4-CLIX
}

COZTICPATLI adiantino

o medicamento amarillo parecido al culantrillo de pozo

Otros autores: Batalla et al. (1942-1943), sugieren que puede ser una especie de Adiantum, por los comentarios de Hernández.

Presente trabajo: Puede tratarse de una ranunculácea del género Thalictrum; esta idea se basa en que Hernández menciona que las raíces tienen sabor amargo y son amarillentas (véase abajo: ixiayáhoal).

\author{
11-XXXVI \\ IXIAYÁHOAL
}

que los mechoacanenses llaman puenga

Otros autores: Paso y Troncoso (1886), sugiere que se parece a Adiantum.

Presente trabajo: La característica de ser “...hierba con raíces largas, amarillas y fibrosas..." sugiere que puede ser una ranunculácea del género Thalictrum (véase VázquezYanes y Pérez-García, 1977). 


\section{DISCUSION}

De la revisión de la obra de Francisco Hernández, Historia de las Plantas de Nueva España, se desprende que según el mismo Hernández y otros revisores de la obra, cincuenta y dos plantas de las más de tres mil mencionadas son pteridofitas, ya sea porque el autor las llama "helechos" o porque en la descripción se incluyen términos que se refieren a nombres comunes en latín o en español y que son aplicados a pteridofitas, como se verá más adelante o porque, en la mínima parte de los casos, la ilustración así nos lo indica.

Las características del análisis de cada planta nos permiten separar a aquellas incluidas en este estudio en tres grupos:

a) Cinco de ellas, de acuerdo con los datos proporcionados en las descripciones $y$ en opinión de los autores de este estudio, no son pteridofitas, sino angiospermas, probablemente una coriariácea (3-XVIII), una piperácea $(3-\mathrm{CXCI})$, dos ranunculáceas del género Thalictrum (2-XXXVI y 4-CLIX) y cabe mencionar que en este caso los folíolos de la angiosperma y los últimos segmentos de la hoja del helecho son muy similares (Vázquez-Yanes y Pérez-Garcia, 1977) y de aquí la confusión, que se aclara cuando Hernández hace mención del sabor amargo y del color amarillo de las raíces de las plantas, característico de las especies de Thalictrum y, por último, en este grupo cabe una planta desconocida (1-LVI).

b) Otro grupo lo integran descripciones de doce plantas que según Hernández son helechos; otros autores indican que cuatro de ellas pueden ser especies de Polypodium, pero nosotros no encontramos evidencias suficientes para interpretarlas como tales. Las ocho plantas restantes probablemente sean helechos, pero no hay datos necesarios para su correcta identificación.

c) Las restantes treinta y cinco plantas pensamos que si son susceptibles de interpretación, cuando menos a nivel genérico, como se menciona a continuación. En doce de ellas hay coincidencia entre las opiniones de los autores de revisiones anteriores y la opinión de nosotros, con las actualizaciones taxonómicas y nomenclaturales necesarias ( $p$. ej., Phyllitis nigripes (Fée) O. Ktze. = Schaffneria nigripes Fée); en dos casos, aunque la descripción se refiere a helechos, la escasez de datos no permite ninguna interpretación. Hay diferencias de opinión en la interpretación de seis plantas.

Para el análisis de las quince plantas restantes y que no han sido interpretadas por otros autores, nos hemos basado principalmente en los nombres y rasgos que Hernández desliza en las descripciones (aunque tomados con cierta reserva) y en la comparación con ejemplares de herbario. Algunos de estos términos son los siguientes:

"lengua de ciervo": por la definición que se tiene de este término en el Diccionario de la Real Academia Española (1941), es muy probable que se trate de alguna especie europea de Phyllitis y creemos que lo que Hernández llama de esta manera deben ser helechos aspleniáceos de hoja simple. 
Riba et al.: Las Pteridofitas en la Historia de las Plantas de la Nueva España

"culantrillo de pozo": no hay la menor duda de que se refiere el autor a Adiantum capillus-veneris o a alguna especie similar. Bajo el nombre de "culantrillo" deben agregarse especies de Pellaea e, incluso, algunas de Notholaena.

"capilares": con tal término Hernández aparentemente cubre géneros muy disímiles, por la semejanza de las "raíces cabelludas como el culantrillo de pozo (Adiantum spp.), la lengua de ciervo (Phyllitis spp.), el helecho (?), el polipodio (Polypodium spp.)...." (Libro 1 , capítulo I).

"Ionquite aspero, mayor o menor": aunque hay un género Lonchitis entre los helechos, éste sólo tiene dos especies, una africana y otra neotropical. En la literatura se señala que "lonchités" es un nombre usado por Dioscórides para cierta planta. En información proporcionada por Lora González (com. pers.), se dice que el término "lonquite" - "lonchite", de origen astur, es el nombre vernáculo de Blechnum spicant ssp. spicant en la región donde éste crece en España; es probable, por lo tanto que las plantas a las que Hernández llama "Ionquite áspero mayor o menor", sean especies americanas de este género. Sin embargo, quedan dudas en aquellos casos en los que la morfología general de la hoja de algunos géneros de helechos es similar, por ejemplo, Polypodium-BlechnumPhanerophlebia especies 1-pinnadas de Asplenium y Diplazium; Elaphoglossum-PleopeltisMicrogramma y otras.

La identidad de una gran parte de las plantas mencionadas por Hérnández en su obra, de cualquier manera seguirá siendo desconocida por la falta de datos y la desaparición de material original; en el caso particular de los helechos, no son muy usados en la herbolaria tradicional de México y es muy probable que sus propiedades curativas hayan sido más supuestas que reales, sin que por ello disminuya su contribución en el aspecto psicológico de las curaciones en las que eran utilizadas. En apoyo de este punto, es significativo que en el Libellus de Medicinalibus Indorum Herbis, más conocido como el Códice de la Cruz-Badiano (1964), se incluye una sola representación gráfica que probablemente sea una pteridofita del género Selaginella.

\section{AGRADECIMIENTOS}

Los autores agradecen al Biól. Javier Valdés y a los revisores anónimos la lectura y revisión del manuscrito así como sus valiosos comentarios. Las fotografías fueron tomadas por Jorge Lodigiani.

\section{LITERATURA CITADA}

Anónimo (diversos autores). s. fecha. Apuntes inéditos. Plantas Medicinales. Archivo histórico de la biblioteca del Departamento de Botánica del Instituto de Biología, Universidad Nacional Autónoma de México. México, D. F. 18 vols.

Anónimo (diversos autores). s. fecha. Apuntes inéditos. Plantas Mexicanas. Archivo histórico de la biblioteca del Departamento de Botánica del Instituto de Biología, Universidad Nacional Autónoma de México. México, D. F. 3 vols. 
Anónimo. 1941. Diccionario de la lengua española. Real Academia Española. Madrid.

Batalla, M. A., D. Ramírez Cantú e I. Rivera Morales (comentaristas botánicos). 1942-1943. In: Hemández, F. Historia de las plantas de Nueva España. Imprenta Universitaria, México, D. F. vols. 1 y 2.

De la Cruz, M. 1964. Libellus de medicinalibus indorum herbis. Manuscrito azteca de 1552. Según traducción latina de Juan Badiano. Instituto Mexicano del Seguro Social. México, D. F. 394 pp.

Flores Olvera, H. y J. Valdés Gutiérrez. 1979. La obra botánica del Dr. Francisco Hemández a más de cuatrocientos años de su realización. Medicina Tradicional 2(6): 84-88.

Hemández, F. 1959. Historia de las plantas de la Nueva España. In: Obras completas. Universidad Nacional Autónoma de México. Imprenta Universitaria. México, D. F. vols. 2 y 3.

Martínez, M. 1979. Catálogo de nombres vulgares y científicos de plantas mexicanas. Fondo de Cultura Económica. México, D. F. 1222 pp.

Miranda, F., M. A. Batalla, D. Ramírez Cantú e I. Rivera Morales (comentaristas botánicos). 1946. In: Hernández, F. Historia de las plantas de Nueva España. Imprenta Universitaria. México, D. F. vol. 3.

Miranda, F. y J. Valdés. 1964. Comentarios botánicos. In: De la Cruz, M. Libellus de medicinalibus indorum herbis. Instituto Mexicano del Seguro Social Méx. Edición facsimilar. pp. 243-284.

Paso y Troncoso, F. del. 1886. Estudios sobre la historia de la medicina en México. I. La botánica entre los nahuas. An. Mus. Nac. México 3: 137-235.

Somolinos D'Ardois, G. 1960. Vida y obra de Francisco Hernández. In: Hernández, F. Obras Completas. Universidad Nacional Autónoma de México, México D. F. vol. 1: 95-373.

Valdés, J. y H. Flores. 1984 [1985]. Comentarios a la obra de Francisco Hernández. In: Hemández, F. 1984 [1985]. Obras completas, v. 7. Universidad Nacional Autónoma de México. Imprenta Universitaria. México, D. F. vol. 7. 222 pp.

Vázquez-Yanes, C. y B. Pérez-García. 1977. Ecological and morphological similarities between Adiantum poiretii Wikstr. and Thalictrum stipitatum Rose. Amer. Fem J. 67(3): 96. 